\title{
Adesão à terapêutica medicamentosa e fatores associados em Diabetes Mellitus tipo 2
}

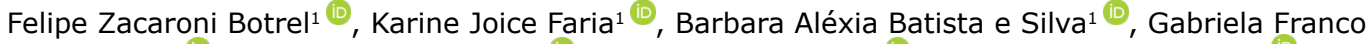 \\ Nascimento $^{1}$, Mariana Moreira Diniz ${ }^{1}{ }^{\mathbb{D}}$, Aisha Aguiar Morais ${ }^{1}$, Daniel Nogueira Cortez ${ }^{1}$
}

\begin{abstract}
RESUMO
Introdução: o Diabetes Mellitus tipo 2 (DM2) é condição crônica que frequentemente cursa com baixa adesão ao tratamento. Objetivo: o presente estudo avaliou a prevalência de adesão à terapêutica medicamentosa e fatores associados em pessoas com DM2 assistidas pela Estratégia Saúde da Família (ESF). Método: trata-se de estudo observacional transversal realizado na ESF da zona urbana de um município de médio porte de Minas Gerais, com amostra randômica de 190 participantes. Foram utilizados dois instrumentos validados: Medida de Adesão ao Tratamento (MAT), que avalia o uso diário de medicamentos prescritos, e teste de Batalla, que avalia adesão através do conhecimento em DM2. O perfil socioeconômico e clínico foi coletado por questionário elaborado pelos autores. Principais resultados: a adesão avaliada pelo MAT $(84,2 \%)$ se contrapôs àquela estimada pelo teste de Batalla (44,2\%). Maiores valores apresentados pelo MAT obtiveram associação significativa com polifarmácia (OR=2,7; IC $95 \%=1,2-6,1)$. Melhor adesão pelo teste de Batalla obteve associação com idade abaixo de 60 anos (OR=3,7; IC $95 \%=1,9-7,1)$, presença de companheiro (OR=2,2; IC 95\%=1,1-4,1), associação de antidiabético oral à insulina $(\mathrm{OR}=2,2$; IC $95 \%=1,2-4,0)$ e cumprimento da meta de atividade física $(\mathrm{OR}=2,6$; IC 95\%=1,2-5,6). Conclusão: os índices de adesão ao uso diário de medicamentos e conhecimento em DM2 verificados pelo estudo são concordantes com a literatura. Piores resultados presentes na população idosa e sem companheiro evidenciam grupo vulnerável que deve receber especial atenção das equipes de saúde.
\end{abstract}

Palavras-chave: Cooperação e adesão ao tratamento, Diabetes Mellitus tipo 2, Conhecimento, Autocuidado. 


\section{INTRODUÇÃO}

O Diabetes Mellitus tipo 2 (DM2) é uma condição crônica cujo tratamento impõe a reorganização do estilo de vida, no que diz respeito aos hábitos alimentares, à prática de atividade física e ao uso de medicamentos ${ }^{1}$.

A baixa adesão ao tratamento do DM2 é problema reconhecido mundialmente ${ }^{2}$. $O$ termo adesão ao tratamento medicamentoso diz respeito à extensão em que o comportamento do paciente corresponde às recomendações prescritas pelo profissional de saúde, no que se refere aos horários, dosagens e frequência de administração dos medicamentos ${ }^{3}$.

A adesão é determinante no curso da doença. Baixa adesão aumenta o risco de complicações e gastos para o sistema de saúde ${ }^{4-6}$. São descritos inúmeros fatores que interferem na adesão. Dentre os fatores que se associam à baixa adesão, destacam-se: baixo nível socioeconômico, hábitos de vida sedentários, doença assintomática e crônica, esquemas terapêuticos complexos e inadequação da relação médico-paciente ${ }^{4,7}$.

Por outro lado, educação em diabetes, maior escolaridade, consolidação do autocuidado apoiado e presença de rede de apoio são apontados como alternativas que contribuem para melhorar os níveis de adesão ${ }^{2}$. Nesse contexto, percebem-se como essenciais as iniciativas da Estratégia de Saúde da Família (ESF) para promoção da saúde.

A OMS enfatiza a necessidade da realização de pesquisas acerca da adesão terapêutica nos países em desenvolvimento, pois é neles que se espera maior aumento na incidência e na prevalência das condições crônicas, como o DM2².

O objetivo deste estudo foi, portanto, avaliar a adesão à terapia medicamentosa e fatores associados em DM2 na ESF.

\section{MÉTODO}

Trata-se de estudo observacional transversal, realizado nas ESF da zona urbana de município de médio porte localizado na região centro-oeste do estado de Minas Gerais, Brasil. A população do estudo foi constituída por 2.978 pacientes com Diabetes Mellitus (DM) cadastrados nas 32 ESF do município, que correspondem à cobertura de $47,4 \%$ da população $0^{8,9}$.

Considerando o nível de significância de $95 \%$ e $84,4 \%$ como prevalência de adesão medicamentosa encontrado em estudo com metodologia e população similares ${ }^{7,10}$, calculou-se uma amostra de 190 usuários $^{10}$.

Os participantes foram elencados de forma proporcional ao número de diabéticos por região de planejamento em saúde da zona urbana do município ${ }^{11}$.
A cidade é administrativamente organizada em nove regiões. Apenas oito áreas contavam com o modelo de ESF para atendimento da população.

Uma ESF em cada região foi definida de forma randômica. Os pacientes da ESF sorteada foram listados e contactados de maneira aleatória até que o tamanho amostral da região fosse contemplado. Em caso de esgotamento do número de pacientes, sem que o número mínimo esperado fosse atingido, uma nova ESF foi sorteada naquela região. Foram selecionadas 11 ESF.

Os participantes foram recrutados entre outubro de 2018 e abril de 2019 e os dados coletados no período de novembro de 2018 a maio de 2019. A busca ativa ocorreu por contato telefônico, em duas tentativas em horários distintos, sempre que necessário.

A visita domiciliar ocorreu em data e hora agendados dentro da disponibilidade dos pesquisadores. $\mathrm{Na}$ impossibilidade de contato, incompatibilidade de agenda ou recusa, a busca passou ao próximo participante, no intuito de garantir que o tamanho amostral fosse contemplado. Foram realizadas 2.391 ligações para a seleção dos 190 participantes. Como perda, 2.201 usuários deixaram de participar da pesquisa sendo 915 devido à desatualização do número telefônico, 1.056 por não resposta ao telefonema e 230 por recusa.

A aplicação dos questionários ocorreu por entrevista, realizada após explicação sobre a pesquisa e assinatura do Termo de Consentimento Livre e Esclarecido. A realização do projeto-piloto permitiu refinar o questionário e sua aplicação pelos pesquisadores. Os participantes que apresentaram sinais e/ou sintomas clássicos de hiperglicemia (poliúria, polipsia, polifagia ou perda de peso não intencional) referidos no momento da coleta ou que detinham exames laboratoriais demonstrando ausência de bom controle glicêmico, receberam relatório destinado à ESF descrevendo seu caso e sua necessidade de atendimento sem delongas. Nenhum paciente necessitou de atendimento de caráter urgente.

Foram critérios de inclusão: estar cadastrado no Sistema de Informação de Saúde (SIS) do município com DM, ter idade mínima de 20 anos, correspondente à faixa etária disponível no SIS para estratificação da população com maior prevalência do DM2. Foram critérios de exclusão: diagnóstico autodeclarado de Diabetes Mellitus Tipo 1 ou outros tipos de DM e impossibilidade de contato telefônico, incompatibilidade de horário e incapacidade de comunicação.

A adesão à terapêutica medicamentosa foi avaliada por dois testes validados para uso no Brasi ${ }^{12}$ : Medida de Adesão ao Tratamento (MAT) e o teste de Batalla. O MAT é instrumento que avalia a adesão ao tratamento medicamentoso prescrito. Para tanto, a receita médica foi conferida. Constitui-se de 
sete perguntas, respondidas em uma escala de Likert que varia entre um (sempre) e seis (nunca). Foram considerados aderentes aqueles participantes que obtiveram um resultado maior ou igual a cinco, o que corresponde à média das respostas raramente ou nunca ${ }^{13}$.

O teste de Batalla mensura a adesão à terapêutica medicamentosa através do conhecimento do participante sobre sua condição. Consiste em três perguntas: 1. O DM é uma doença para vida toda? 2. O DM pode ser controlado com dieta e medicamentos? 3. Cite dois ou mais órgãos afetados pelo DM. Participantes que responderam corretamente a todas as perguntas foram considerados aderentes ${ }^{12,14}$.

Os fatores associados à adesão foram obtidos por questionário estruturado elaborado pelos autores a partir de revisão bibliográfica. Esse, baseia-se nos principais fatores associados à adesão terapêutica como: sexo, idade, situação conjugal, escolaridade, tempo de diagnóstico, prática e cumprimento das recomendações sobre atividade física, terapia medicamentosa e polifarmácia (uso diário de cinco ou mais medicamentos) 4,7,15.

Como prática de atividade física, foi considerado qualquer exercício praticado com finalidade terapêutica. A recomendação mínima de exercício utilizada foi de 150 minutos semanais divididos em pelo menos três dias ${ }^{16}$. Foram pesquisadas sete complicações autodeclaradas do DM2: neuropatia, doença arterial coronariana, retinopatia, infarto agudo do miocárdio, acidente vascular encefálico, doença renal crônica e amputação não traumática.

Os participantes foram categorizados conforme renda média domiciliar, através do Critério de Classificação Econômica Brasil ${ }^{17}$.

A associação entre as condições clínicas, assistenciais e socioeconômicas (variáveis de exposição), e a adesão ao tratamento avaliada pelo MAT e pelo teste de Batalla (variáveis de desfecho) foi realizada pelo teste de Qui-Quadrado de Pearson. As categorias que obtiveram valor $p<0,20$ foram inseridas pelo método backward no modelo multivariado de regressão logística múltipla. Utilizou-se, para verificação do ajuste do modelo final, o teste de Hosmer \& Lemeshow. A razão de chances - odds ratio (OR) com intervalo de confiança de $95 \%$ (IC 95\%) foi utilizada como medida de efeito. Para todas as análises, foi adotado o valor de significância estatística de $5 \%(p<0,05)$. As informações foram armazenadas em planilha do MSExcel 2016 e analisadas com apoio do programa Statistical Package for the Social Sciences $®$ versão 20.0 .

O projeto foi devidamente aprovado pelo Comitê de Ética em Pesquisa com Seres Humanos (CAAE: 80092717.7.0000.5545).

\section{RESULTADOS}

Em relação à caracterização socioeconômica, a população do estudo foi composta majoritariamente por mulheres. A idade média foi de 65,5 anos. Aproximadamente dois terços da população vivia com companheiro, $73,7 \%$ estudou até o ensino fundamental incompleto e $58,9 \%$ possuía renda média domiciliar entre $R \$ 1.625,00$ e $R \$ 2.705,00$ (Tabela 1).

Tabela 1. Distribuição percentual das variáveis clínicas e aspectos sociodemográficos e econômicos.

\begin{tabular}{|c|c|}
\hline Variáveis $(n=190)$ & n (\%) \\
\hline Sexo Feminino & $120(63,2 \%)$ \\
\hline Sexo Masculino & $70(36,8 \%)$ \\
\hline Sem companheiro(a) & $63(33,2 \%)$ \\
\hline Com companheiro(a) & $127(66,8 \%)$ \\
\hline \multicolumn{2}{|l|}{ Escolaridade } \\
\hline Analfabeto / EF Incompleto & $140(73,7 \%)$ \\
\hline EF Completo ou mais & $50(26,3 \%)$ \\
\hline \multicolumn{2}{|l|}{ Renda Média Domiciliar } \\
\hline$R \$ 768,00$ & $48(25,3 \%)$ \\
\hline$R \$ 1.625,00-R \$ 2.705,00$ & $112(58,9 \%)$ \\
\hline$R \$ 4.852,00-R \$ 20.888,00$ & $30(15,8 \%)$ \\
\hline \multicolumn{2}{|l|}{ Atividade Física } \\
\hline Sedentário & $121(63,7 \%)$ \\
\hline Não sedentário & $69(36,3 \%)$ \\
\hline Cumpre recomendação & $43(22,6 \%)$ \\
\hline Não cumpre recomendação & $26(13,7 \%)$ \\
\hline \multicolumn{2}{|l|}{ Tabagismo } \\
\hline Nunca fumou & $106(55,8 \%)$ \\
\hline Ex-tabagista & $66(34,7 \%)$ \\
\hline Tabagista & $18(9,5 \%)$ \\
\hline \multicolumn{2}{|l|}{ Etilismo } \\
\hline Sim & $148(77,9 \%)$ \\
\hline Não & $42(22,1 \%)$ \\
\hline \multicolumn{2}{|l|}{ Tempo de Diagnóstico } \\
\hline$<5$ anos & $28(14,7 \%)$ \\
\hline$\geq 5$ a $<10$ anos & $57(30,0 \%)$ \\
\hline$\geq 10$ anos & $105(55,3 \%)$ \\
\hline \multicolumn{2}{|l|}{ Presença de Complicações } \\
\hline Sim & $136(71,6 \%)$ \\
\hline Não & $54(28,4 \%)$ \\
\hline \multicolumn{2}{|l|}{ Terapia Medicamentosa } \\
\hline Apenas ADO & $113(59,5 \%)$ \\
\hline Apenas Insulina & $18(9,5 \%)$ \\
\hline \multicolumn{2}{|l|}{ Associação ADO + Insulina } \\
\hline $\begin{array}{l}\text { Não utiliza terapia } \\
\text { medicamentosa }\end{array}$ & $54(28,4 \%)$ \\
\hline \multicolumn{2}{|l|}{$5(2,6 \%)$} \\
\hline \multicolumn{2}{|l|}{ Polifarmácia } \\
\hline Sim & $137(72,1 \%)$ \\
\hline Não & $53(27,9 \%)$ \\
\hline
\end{tabular}


Foi encontrada taxa de adesão avaliada pelo MAT de $84,2 \%$, enquanto pelo teste de Batalla o valor foi $44,2 \%$. Cerca de um terço dos entrevistados foi considerado aderente por ambos os métodos. Dentre os participantes considerados aderentes pelo Batalla, $86,9 \%$ eram também aderentes ao MAT, porém, essa associação pode dever-se ao acaso.

Pela avaliação do MAT, os principais responsáveis pela não adesão foram o esquecimento de tomadas diárias e o descuido com horário das medicações. A maior dificuldade apresentada no teste de Batalla foi citar pelo menos dois órgãos afetados pelo DM2. Apenas $50 \%$ souberam responder corretamente essa pergunta.

A principal classe de ADO utilizada foi biguanida, presente na prescrição de $82,6 \%$ dos participantes. Em média, eram utilizados 6,17 medicamentos ao dia. Dentre aqueles em polifarmácia, $66,5 \%$ viviam com companheiro e $63,5 \%$ possuíam pelo menos o Ensino Fundamental (EF) completo.

No que se refere às complicações do DM2 pesquisadas, $70 \%$ dos participantes apresentava alguma complicação/lesão em órgão-alvo. A neuropatia foi a mais frequente $(48,9 \%)$, seguida por doença arterial coronariana $(25,2 \%)$, retinopatia $(20,5 \%)$, infarto agudo do miocárdio $(10 \%)$, acidente vascular encefálico $(7,9 \%)$, doença renal crônica $(7,9 \%)$ e amputação não traumática $(5,3 \%)$.

Pela análise univariada dos resultados, apenas a variável polifarmácia apresentou associação significativa com a adesão à terapia medicamentosa medida pelo MAT (Tabela 2). Esta associação mantevese após regressão logística múltipla e permitiu concluir que os participantes que apresentaram polifarmácia tiveram 2,7 vezes mais chances de serem aderentes à terapêutica medicamentosa.

Quanto ao resultado do Batalla, foi encontrada associação estatisticamente significativa quanto à idade, situação conjugal e uso de ADO associado à insulina (Tabela 3). Após regressão logística, a variável cumprir recomendação de atividade física passou a ser significativa e a situação conjugal não se sustentou.

Pela regressão logística realizada com o teste de Batalla, os participantes com menos de 60 anos tiveram 4,4 vezes mais chances de serem considerados aderentes. Aqueles que cumpriram a recomendação de atividade física evidenciaram 2,6 vezes mais chances de apresentar adesão terapêutica por meio do conhecimento. Da mesma forma, os participantes que associavam ADO à insulina apresentaram 2,9 vezes mais chances de melhor resultado.

\section{DISCUSSÃO}

O presente estudo avaliou os índices de adesão à terapêutica medicamentosa para DM2 e seus fatores associados. A adesão terapêutica avaliada através do uso diário de medicamentos (MAT) mostrou-se superior àquela avaliada pelo conhecimento em DM2 (teste de Batalla) $(84,2 \%$ versus $44,2 \%)$. O uso regular dos medicamentos prescritos foi mais comum nos pacientes em polifarmácia. Os grupos que demonstraram maior conhecimento em diabetes foram os de idade inferior a 60 anos, com companheiro, em uso concomitante de ADO e insulina e aderentes à recomendação de atividade física.

Foram considerados aderentes pelo MAT, 84,2\% dos participantes. Estudos nacionais, com metodologia similar, obtiveram resultados semelhantes: $78,3 \%$, $84,4 \%$ e $95,7 \% .7,15,18$. Em uma revisão sistemática, os valores de adesão à terapia medicamentosa variaram entre $36-93 \%^{19}$. As maiores taxas de adesão ao tratamento medicamentoso em comparação aos outros pilares terapêuticos, pode se dever à importância conferida a ele pelos pacientes e ao pior acesso aos meios necessários à mudança do estilo de vida 715,20

O estudo demonstrou que indivíduos em polifarmácia tendem a ser mais aderentes ao tratamento medicamentoso. A literatura diverge quanto ao impacto da polifarmácia na adesão ${ }^{2,15,21,22}$. Apesar desta condição estar relacionada à ocorrência de efeitos adversos e ao aumento da frequência de tomada de medicamentos, ela não necessariamente resulta em pior adesão ${ }^{15,21}$.

É relevante salientar que dentre os participantes em polifarmácia, foi frequente a escolaridade com EF completo. Este resultado reforça a hipótese de que a presença de maior tempo de estudo pode influenciar positivamente a adesão ${ }^{15,21}$. Tal condição combinada ao significado que o paciente atribui à terapia medicamentosa pode motivá-lo a aderir ao tratamento, apesar das dificuldades inerentes à polifarmácia.

Outra hipótese diz respeito à presença de maior número de complicações crônicas decorrentes do DM2 em pacientes com polifarmácia. Pacientes com doença mais grave, muitas vezes sintomática, apresentariam melhores índices de adesão, em contraponto àqueles com doença inicial e assintomática ${ }^{21}$.

Níveis de adesão pelo conhecimento em DM2 abaixo do desejado foram encontrados (44,2\%), em concordância com outros estudos ${ }^{4,27}$. A baixa adesão verificada pelo teste de Batalla reflete a carência de conhecimento em DM2 pelos pacientes ${ }^{2,13}$. Estudo prévio relacionou baixo conhecimento a poucos anos de estudo completos e menor participação 
Tabela 2. Associação entre MAT e as variáveis clínicas, aspectos sociodemográfi-cos e econômicos.

\begin{tabular}{|c|c|c|c|c|c|}
\hline Variáveis $(n=190)$ & Aderente $n(\%)$ & Não Aderente $n$ (\%) & OR & IC $(95 \%)$ & $\boldsymbol{p}$ \\
\hline \multicolumn{6}{|l|}{ Batalla } \\
\hline Aderente & $73(86,9)$ & $11(13,1)$ & 1,4 & $0,6-3,2$ & 0,365 \\
\hline Não Aderente & $87(82,1)$ & $19(17,9)$ & & 1 & \\
\hline Sexo Feminino & $100(83,3)$ & $20(16,7)$ & 0,8 & $0,4-1,9$ & 0,664 \\
\hline Sexo Masculino & $60(85,7)$ & $10(14,3)$ & & 1 & \\
\hline \multicolumn{6}{|l|}{ Idade } \\
\hline$<60$ anos & $44(81,5)$ & $10(18,5)$ & 0,7 & $0,3-1,7$ & 0,516 \\
\hline$\geq 60$ anos & $116(85,3)$ & $20(14,7)$ & & 1 & \\
\hline Sem Companheiro(a) & $53(84,1)$ & $10(15,9)$ & 1,0 & 1 & 0,982 \\
\hline Com Companheiro(a) & $107(84,3)$ & $20(15,7)$ & & $0,4-2,3$ & \\
\hline \multicolumn{6}{|l|}{ Escolaridade } \\
\hline Analfabeto / EF Incompleto & $50(79,4)$ & $13(20,6)$ & 1,7 & 1 & 0,197 \\
\hline EF Completo ou mais & $110(86,6)$ & $17(13,4)$ & & $0,7-3,7$ & \\
\hline \multicolumn{6}{|l|}{ Renda Média Domiciliar } \\
\hline$R \$ 768,00-R \$ 2.705,00$ & $135(84,4 \%)$ & $25(15,6 \%)$ & 0,9 & 1 & 0,886 \\
\hline$R \$ 4.852,00-R \$ 20.888,00$ & $25(83,3 \%)$ & $5(16,7 \%)$ & & $0,3-2,6$ & \\
\hline \multicolumn{6}{|l|}{ Atividade Física } \\
\hline Sedentário & $100(82,6)$ & $21(17,4)$ & 1,4 & 1 & 0,433 \\
\hline Não sedentário & $60(87,0)$ & $9(13,0)$ & & $0,6-3,2$ & \\
\hline Cumpre recomendação & $35(81,4)$ & $8(18,6)$ & 0,8 & $0,3-1,9$ & 0,565 \\
\hline Não cumpre recomendação & $125(85,0)$ & $22(15,0)$ & & 1 & \\
\hline \multicolumn{6}{|l|}{ Tabagismo } \\
\hline Nunca fumou & $88(83,0)$ & $18(17,0)$ & 0,9 & $0,2-3,7$ & 0,974 \\
\hline Ex-tabagista & $57(86,4)$ & $9(13,6)$ & 1,2 & $0,3-5,3$ & 0,745 \\
\hline Tabagista & $15(83,3)$ & $3(16,7)$ & & 1 & \\
\hline \multicolumn{6}{|l|}{ Etilismo } \\
\hline Sim & $20(80)$ & $5(20)$ & & 1 & \\
\hline Não & $140(84,8)$ & $25(15,2)$ & 1,4 & $0,5-4,1$ & 0,536 \\
\hline \multicolumn{6}{|l|}{ Tempo de Diagnóstico } \\
\hline$<5$ anos & $21(75)$ & $7(25)$ & & 1 & \\
\hline$\geq 5 \mathrm{a}<10$ anos & $8(14)$ & $49(86)$ & 2,0 & $0,7-6,4$ & 0,213 \\
\hline$\geq 10$ anos & $90(85,7)$ & $15(14,3)$ & 2,0 & $0,7-5,5$ & 0,175 \\
\hline \multicolumn{6}{|l|}{ Presença Complicações } \\
\hline Sim & $11(85,3)$ & $20(14,7)$ & 1,3 & $0,6-3,0$ & 0,516 \\
\hline Não & $44(81,5)$ & $10(18,5)$ & & 1 & \\
\hline \multicolumn{6}{|l|}{ Usa apenas ADO } \\
\hline $\operatorname{Sim}$ & $99(87,6)$ & $14(12,4)$ & 0,5 & 1 & 0,119 \\
\hline Não & $61(79,2)$ & $16(20,8)$ & & $0,2-1,2$ & \\
\hline \multicolumn{6}{|l|}{ Usa apenas insulina } \\
\hline Sim & $14(77,8)$ & $4(22,2)$ & 0,6 & $0,2-2,0$ & 0,431 \\
\hline Não & $146(84,9)$ & $26(15,1)$ & & 1 & \\
\hline \multicolumn{6}{|l|}{ Polifarmácia } \\
\hline Sim & $121(88,3)$ & $16(11,7)$ & 2,7 & $1,2-6,1$ & $0,012 *$ \\
\hline Não & $39(73,6)$ & $14(26,4)$ & & 1 & \\
\hline
\end{tabular}

EF: Ensino Fundamental. ADO: Antidiabético Oral.

* Variáveis com associação estatisticamente significante. 
Tabela 3. Associação entre Batalla e as variáveis clínicas, aspectos sociodemográ-ficos e econômicos.

\begin{tabular}{|c|c|c|c|c|c|}
\hline Variáveis $(n=190)$ & Aderente $n(\%)$ & Não Aderente $n$ (\%) & OR & IC $(95 \%)$ & $\boldsymbol{p}$ \\
\hline Sexo Feminino & $34(48,6)$ & $36(51,4)$ & 0,7 & $0,4-1,4$ & 0,355 \\
\hline Sexo Masculino & $50(41,7)$ & $70(58,3)$ & & 1 & \\
\hline \multicolumn{6}{|l|}{ Idade } \\
\hline$<60$ anos & $36(66,7)$ & $18(33,3 \%)$ & 3,7 & $1,9-7,1$ & $<0,001^{*}$ \\
\hline$\geq 60$ anos & $48(35,3)$ & $88(64,7 \%)$ & & 1 & \\
\hline Sem Companheiro(a) & $20(31,7)$ & $43(68,3 \%)$ & 2,2 & $1,1-4,1$ & $0,015^{*}$ \\
\hline Com Companheiro(a) & $64(50,4)$ & $63(49,6 \%)$ & & 1 & \\
\hline \multicolumn{6}{|l|}{ Escolaridade } \\
\hline Analfabeto / EF Incompleto & $23(36,5)$ & $40(63,5 \%)$ & & 1 & 0,132 \\
\hline EF Completo ou mais & $61(48,0)$ & $66(52,0 \%)$ & 1,6 & $0,9-2,988$ & \\
\hline \multicolumn{6}{|l|}{ Renda Média Domiciliar } \\
\hline$R \$ 768,00-R \$ 2.705,00$ & $73(45,6 \%)$ & $87(54,4 \%)$ & 0,7 & 1 & 0,365 \\
\hline$R \$ 4.852,00-R \$ 20.888,00$ & $11(36,7 \%)$ & $19(63,3 \%)$ & & $0,3-1,5$ & \\
\hline \multicolumn{6}{|l|}{ Atividade Física } \\
\hline Sedentário & $49(40,5)$ & $72(59,5)$ & 1,5 & $0,8-2,7$ & 0,172 \\
\hline Não sedentário & $35(50,7)$ & $34(49,3)$ & & 1 & \\
\hline Cumpre recomendação & $23(53,5)$ & $20(46,5)$ & 1,6 & $0,8-3,2$ & 0,164 \\
\hline Não cumpre recomendação & $61(41,5)$ & $86(58,5)$ & & 1 & \\
\hline \multicolumn{6}{|l|}{ Tabagismo } \\
\hline Nunca fumou & $48(45,3)$ & $58(54,7)$ & 1,3 & $0,5-3,6$ & 0,614 \\
\hline Ex-tabagista & $29(43,9)$ & $37(56,1)$ & 1,2 & $0,4-3,6$ & 0,701 \\
\hline Tabagista & $7(38,9)$ & $11(61,1)$ & & 1 & \\
\hline \multicolumn{6}{|l|}{ Etilismo } \\
\hline Sim & $15(60,0)$ & $10(40,0)$ & & 1 & \\
\hline Não & $69(41,8)$ & $96(58,2)$ & 0,5 & $0,2-1,1$ & 0,088 \\
\hline \multicolumn{6}{|l|}{ Tempo de Diagnóstico } \\
\hline$<5$ anos & $11(39,3)$ & $17(60,7)$ & & 1 & \\
\hline$\geq 5$ a $<10$ anos & $23(40,4)$ & $34(59,6)$ & 1,0 & $0,4-2,6$ & 0,925 \\
\hline$\geq 10$ anos & $90(85,7)$ & $15(14,3)$ & 1,4 & $0,6-3,3$ & 0,432 \\
\hline \multicolumn{6}{|l|}{ Presença Complicações } \\
\hline Sim & $61(44,9)$ & $75(55,1)$ & 1,1 & $0,6-2,1$ & 0,777 \\
\hline Não & $23(42,6)$ & $31(57,4)$ & & 1 & \\
\hline \multicolumn{6}{|l|}{ Usa apenas ADO } \\
\hline Sim & $41(36,3)$ & $72(63,7)$ & 2,2 & 1 & $0,008^{*}$ \\
\hline Não & $43(55,8)$ & $34(44,2)$ & & $1,2-4,0$ & \\
\hline \multicolumn{6}{|l|}{ Usa apenas insulina } \\
\hline $\operatorname{Sim}$ & $9(50,0)$ & $9(50,0)$ & 1,3 & $0,5-3,4$ & 0,603 \\
\hline Não & $75(43,6)$ & $97(56,4)$ & & 1 & \\
\hline \multicolumn{6}{|l|}{ Polifarmácia } \\
\hline $\operatorname{Sim}$ & $62(45,3)$ & $75(54,7)$ & 1,2 & $0,6-2,2$ & 0,641 \\
\hline Não & $22(41,5)$ & $31(58,5)$ & & 1 & \\
\hline
\end{tabular}

EF: Ensino Fundamental. ADO: Antidiabético Oral.

* Variáveis com associação estatisticamente significante. 
em atividades educativas, corroborando com o encontrado ${ }^{24}$. É alarmante que, enquanto $70 \%$ dos participantes apresentavam alguma complicação crônica do DM2, apenas $50 \%$ soubessem citar pelo menos um órgão-alvo da condição.

Tal escassez de conhecimento observada é particularmente preocupante, tendo em vista que a pesquisa foi realizada exclusivamente com usuários de ESF. É papel da ESF estimular e fomentar o autocuidado apoiado ${ }^{28}$. A baixa cobertura municipal de ESF no presente estudo pode levar a prever um déficit ainda maior na educação em saúde do município como um todo ${ }^{29}$.

Pacientes com menos de 60 anos e vivendo com companheiro apresentaram melhores índices de adesão. A associação negativa da idade avançada com o conhecimento sobre a doença é descrita na literatura e provavelmente se explica pelas alterações cognitivas e funcionais prevalentes nos idosos, que prejudicam a compreensão sobre a doença e seu tratamento 22,24 . Em contraponto, a presença de companheiro representa incentivo ao autocuidado, o que pode ter ampliado a motivação dos participantes para conhecer sua doença e seu tratamento ${ }^{21}$.

A administração concomitante de ADO e insulina também se associou a maior adesão. $O$ emprego da insulina no tratamento do DM2, bem como o aparecimento de complicações, tende a ser tardio a depender do bom controle metabólico ${ }^{16}$. Apesar das dificuldades inerentes ao uso da insulina, como o manejo para sua aplicação e o próprio estigma, seu uso não foi um limitante para adesão ${ }^{2,5}$. A presença de doença mais grave e sintomática associada ao tempo de convívio com a condição pode, mais uma vez, justificar a maior adesão medida através do conhecimento $0^{4,7,20,25}$.

A respeito da prática de atividade física, o resultado do estudo também está em consonância com a literatura. No tratamento do DM2, são esperados índices mais baixos de adesão ao plano alimentar e à atividade física quando comparados aos de uso de medicamentos ${ }^{7,16}$.

Associação foi estabelecida entre o cumprimento da recomendação de atividade física e a adesão pelo teste de Batalla. Estudo prévio demonstrou que participantes que superaram as dificuldades e implementaram atividade física em sua rotina da maneira recomendada, desenvolveram habilidades e atitudes positivas, que se relacionam diretamente com a aquisição de conhecimento sobre a doença e autocuidado ${ }^{6}$.
A ausência de associação significativa entre adesão avaliada pelo MAT e a maioria das variáveis elencadas estava prevista na literatura ${ }^{7,23}$. A ausência de associação com o teste de Batalla reforça a hipótese de que o conhecimento nem sempre é suficiente para sustentar a adesão, que também é atrelada a fatores psicossociais ${ }^{24}$.

Estudos demonstram dificuldade em medir a adesão mesmo pelos melhores instrumentos atualmente disponíveis $^{12}$. O MAT, por exemplo, é instrumento baseado na autodeclaração e depende da veracidade das informações fornecidas, uma limitação do estudo.

Outro obstáculo consiste em mensurar o impacto de todos os aspectos que interferem na adesão em um estudo transversal. A característica metodológica não fornece dados da incidência da adesão medicamentosa, nem associação de causa e efeito nas análises realizadas ${ }^{18,25,26}$.

Dentre as limitações do estudo, a ausência de análise dos níveis de hemoglobina glicada impossibilita a verificação laboratorial do cumprimento da meta glicêmica. A adesão diz respeito ao uso dos medicamentos em um período limitado. A metodologia adotada impede a avaliação dos níveis de persistência ao tratamento, apesar dos níveis de adesão.

\section{CONCLUSÃO}

O presente estudo identifica fragilidades na adesão ao tratamento medicamentoso do DM2 em usuários de ESFs e delimita subpopulações cuja abordagem causariam maior impacto. A população idosa e aquela sem companheiro, em especial, mereceriam abordagens educativas individuais e em grupo com construção de plano terapêutico singular e fortalecimento do autocuidado apoiado.

A aplicação de instrumentos validados e traduzidos para o português torna os achados do presente estudo comparáveis à literatura sobre o tema. A realização prévia de treinamento dos pesquisadores atuantes no campo e de um projeto-piloto demonstram cuidado metodológico e conferem confiabilidade aos resultados. O presente estudo demonstra uma realidade local com adesão aquém do desejável, seja na declaração do uso de medicamentos ou no conhecimento em diabetes. Diante desse cenário, pretende-se estimular intervenções direcionadas e a mensuração de seu impacto. 


\section{REFERÊNCIAS}

1.American Diabetes Association. Diabetes Care. Clin Interv Aging 2019; 42 (Supl.1).

2.World Health Organization. Adherence to long-term therapies - Evidence for action. Geneva; 2003.

3.Cramer JA, Roy A, Burrell A, Fairchild CJ, Fuldeore $M J$, Ollendorf DA, et al. Medication compliance and persistence: terminology and definitions. Value Health. 2008 Jan-Feb;11(1):44-7. doi: 10.1111/j.15244733.2007.00213.x. PMID: 18237359.

4. Rodrigues FL, Santos MA, Teixeira CRS, Gonela JT, Zanettti ML. Relação entre conhecimento, atitude, escolaridade e tempo de doença em indivíduos com diabetes mellitus. Acta Paul Enferm 2012; 25(2): 284-290.

5.Silva AB, Engroff $P$, Sgnaolin V, Ely LS, Gomes I. Prevalência de diabetes mellitus e adesão medicamentosa em idosos da Estratégia Saúde da Família de Porto Alegre/ RS. Cad Saude Colet 2016; 24(3):308-316.

6.Brasil. Ministério da Saúde. Estratégias para o cuidado da pessoa com doença crônica: diabetes mellitus. Cadernos de Atenção Básica n036. Brasília: Ministério da Saúde; 2013.

7.Faria HTG, Santos MA, Arrelias CCA, Rodrigues FFL, Gonela JT, Teixeira CRS, et al. Adesão ao Tratamento em Diabetes Mellitus em Unidades da Estratégia da Saúde da Família. Rev Esc Enferm USP 2014; 48(2): 257-63.

8.Brasil. Ministério da Saúde. Departamento de Atenção Básica. Portal da Saúde; 2017. Histórico de cobertura das Estratégias de Saúde da Família. [acessado 2017 jun 24]. Disponível em: http://dab.saude.gov.br/ portaldab/historico_cobertura_esf.php.

9.Prefeitura de Divinópolis. Secretaria Municipal de Saúde. Sistema de Informação a Saúde (SIS); 2017. Consulta do total de usuários cadastrados em Divinópolis levantada nos sistemas de informação da SEMUSA.

10. Lwanga S, Lemeshow S. Sampla Size Determination in Health Studies Geneva.Geneva : World Health Organization; 1991.

11. Divinópolis. Plano Diretor Participativo de Divinópolis: Configuração territorial de Divinópolis. FUNEDI/UEMG; 2013.

12.Araújo MFM, Freitas RWJF, Marinho NBP, Alencar AMPG, Damasceno MMC, Zanetti ML. Validation of two methods to evaluate adherence to oral anti-diabetic medication. J Nurs Healthc Chronic Illn 2011; 3(3): 275-282.

13. Delgado $A B$, Lima $M L$. Contributo para a validação concorrente de uma medida de adesão aos tratamentos. Psicologia, Saúde e Doenças 2001; 2(2): 81-100.

14. Batalla C, Blanquer A, Ciurana R, Garcia M, Cases E, Pérez A. Cumplimiento de la prescripción farmacológica ver pacientes hipertensos. Aten Primaria 1994; 11.

15.Gomes-Villas Boas L, Foss-Freitas M, Pace A. Adesão de Pessoas com Diabetes Mellitus tipo 2 ao Tratamento Medicamentoso. Rev Bras Enferm 2014; 67(2): 268-73.
16. Sociedade Brasileira de Diabetes. Diretrizes da Sociedade Brasileira de Diabetes (2019-2020). AC Farmacêutica 2020.

17. Associação Brasileira de Empresas de Pesquisa. Critério de Classificação Econômica Brasil; 2016. [acessado 2016 mai 13]. Disponível em: http://www.abep.org/criterio-brasil.

18. Cortez DN, Macedo MML, Souza DAS, Santos JC, Afonso GS, Reis IA, et al. Evaluating the effectiveness of an empowerment program for self-care in type 2 diabetes: a cluster randomized trial. BMC Public Health 2017; 17:41.

19. Cramer J. A systematic review of adherence with medications for diabetes. Diabetes Care. 2004; 5(27):1218-24.

20. Ferreira S, Castleberry G. A systems thinking perspective of medication adherence for patients with Diabetes Mellitus. Pro1cedia Comput Sci 2019; 153: 218-224.

21. Penaforte K, Araújo S, Fernandes V, Barbosa I, Cestari $\mathrm{V}$, Montenegro JR. Associação entre polifarmácia e adesão ao tratamento farmacológico em pacientes com diabetes. Rev. RENE. 2017; 18(5):631-638.

22. Arruda DCJ, Eto FN, Velten APC, Morelato RL, Oliveira ERA. Fatores associados a não adesão medicamentosa entre idosos de um ambulatório filantrópico do Espírito Santo. Rev Bras Geriatr Gerontol 2015; 18(2): 327-33.

23. Arrelias CC, Faria HT, Teixeira CR, Santos MA, Zanetti ML. Adesão ao tratamento do diabetes mellitus e variáveis sociodemográficas, clínicas e de controle metabólico. Acta Paul Enferm 2015; 28(4): 315-322.

24. Borba A, Arruda I, Marques AP, Leal M, Diniz A. Conhecimento sobre o diabetes e atitude para o autocuidado de idosos na atenção primária à saúde. Cien Saude Colet 2019; 24(1): 125-136.

25. Figueira ALG, Villas Boas LCG, Coelho ACM, Freitas MCF, Pace AE. Intervenções educativas para o conhecimento da doença, adesão ao tratamento e controle do diabetes mellitus. Rev Lat Am Enfermagem 2017; 25:e2863. Available from: https://www.scielo.br/j/rlae/a/nxygX8x Kc9DmKcCDNWg5mnq/?format=pdf\&lan $\mathrm{g}=\mathrm{pt}$.

26. Albuquerque G, Nascimento B, Gracia D, Preisler L, Perna $P$, Silva M. Adesão de hipertensos e diabéticos analfabetos ao uso de medicamento a partir da prescrição pictográfica. Trab Educ Saúde 2016; 14(2): 611-624.

27. Barreto TMAC, Rodrigues LJS, Maciel JC, Sampaio DM, Barreto F. Prevalência de adesão ao tratamento medicamentoso por diabéticos no norte do Brasil. SANARE $2017 ; 16(2): 22-30$

28.Salci MA, Meirelles BHS, Silva DMGV. Educação em saúde para prevenção das complicações crônicas do diabetes mellitus na atenção primária. Esc Anna Nery 2018; 22(1).

29. Essler M, Thumé E, Duro SMS, Tomasi E, Siqueira FCV, Silveira DS, et al. Ações educativas e de promoção da saúde em equipes do Programa Nacional de Melhoria do Acesso e da Qualidade da Atenção Básica, Rio Grande do Sul, Brasil. Epidemiol. Serv. Saúde. 2018; 27(2): 2017389. 


\section{Contribuição dos autores}

1. Elaboração do projeto: FZB, KJF, BABS, GFN, MMD, AAM.

2. Coleta de dados: FZB, KJF, BABS, GFN, MMD.

3. Análise de dados: DNC.

4. Escrita do artigo: FZB, KJF, BABS, GFN, MMD.

5. Revisão crítica: AAM, DNC.

\section{Fontes de financiamento}

O trabalho não contou com fontes de apoio financeiro.

Os autores declaram não haver conflito de interesse

Autor Correspondente:

Felipe Zacaroni Botrel

fzacaroni@hotmail.com

Editor:

Prof. Dr. Marcelo Riberto

Recebido: $17 / 11 / 2020$

Aprovado: $13 / 08 / 2021$ 